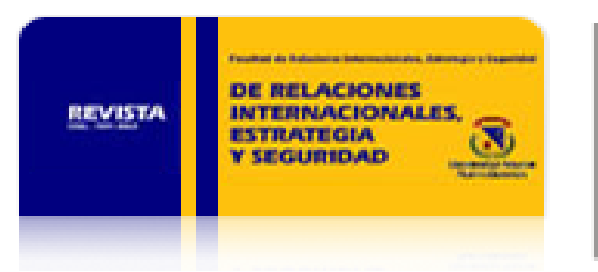

Revista de Relaciones Internacionales, Estrategia y Seguridad

ISSN: 1909-3063

cinuv.relinternal@unimilitar.edu.co

Universidad Militar Nueva Granada

Colombia

Sánchez Cabarcas, Fabio

¿POR QUÉ CONTINÚA SIENDO IMPORTANTE PARA EE.UU. IRAK? GEOESTRATEGIA Y

ENERGÍA EN EL SIGLO XXI

Revista de Relaciones Internacionales, Estrategia y Seguridad, vol. 3, núm. 2, julio-diciembre, 2008,

pp. 161-177

Universidad Militar Nueva Granada

Bogotá, Colombia

Disponible en: http://www.redalyc.org/articulo.oa?id=92712967009

- Cómo citar el artículo

- Número completo

- Más información del artículo

- Página de la revista en redalyc.org

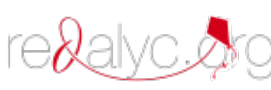

Sistema de Información Científica

Red de Revistas Científicas de América Latina, el Caribe, España y Portugal

Proyecto académico sin fines de lucro, desarrollado bajo la iniciativa de acceso abierto 
rev.relac.int.estrateg.segur.3(2):161-177,2008

\section{¿POR QUÉ CONTINÚA SIENDO IMPORTANTE PARA EE.UU. IRAK? GEOESTRATEGIA Y ENERGÍA EN EL SIGLO XXI}

Fabio Sánchez Cabarcas*

\section{RESUMEN}

Este artículo pretende hacer un diagnóstico de la intervención militar de EE.UU. en Irak desde 2003. En la primera parte se reconstruye el contexto histórico-político en el que la administración Bush ha adelantado tal acción. Posteriormente se analiza la geopolítica de poder de acuerdo a la importancia de los recursos energéticos localizados en Oriente Medio y específicamente en Irak. Luego se analiza la estrategia que EE.UU. a través de la historia ha utilizado en la región, y finalmente se explica por qué ha fracasado la reconstrucción del país, no así la apropiación y explotación de los recursos energéticos del país.

Palabras clave: Conflictos, Irak, geoestrategia, petróleo, Oriente Medio, política exterior, EE.UU.

\footnotetext{
* Comunicador Social-Periodista, PUJ, Magíster en Relaciones Internacionales, PUJ, Candidato a Doctor en Relaciones Internacionales Universidad Autónoma de Barcelona fabiobernardo.sanchez@campus.uab.cat ó fabiosanchezc@yahoo.com.
} 


\begin{abstract}
This paper seeks to make a diagnosis of the U.S. military intervention in Iraq since 2003. In the first section reconstructs the historic and political context under George W. Bush administration. In the second part, the power geopolitics is analyzed according to the importance of the energy resources located in Middle East, specifically in Iraq. Then, American strategy in the region is studied through history. Finally, it's explained why the reconstruction of the country has failed, not so the appropriation and exploitation of the energy resources of the country.
\end{abstract}

Keywords: Conflicts, Iraq, geostrategy, oil, Middle East, foreign policy, U.S.

\title{
INTRODUCCIÓN.
}

Luego de los ataques del 11-s en Nueva York, el gobierno del presidente George W. Bush, elaboró una estrategia política agresiva, que implicaba intervenir en escenarios estratégicos para los intereses económicos y de seguridad de Washington. El plan se basó en la construcción de un «enemigo público» encarnado por AI Qaeda, que fuera suficiente para generar temor y buscar el apoyo de la opinión pública nacional e internacional. De la misma manera, se recrearon una serie de pruebas muy débiles como las supuestas Armas de Destrucción Masiva (ADM) que poseía el régimen de Sadam Hussein, lo cual se desmentiría durante las sesiones del Consejo de Seguridad de Naciones Unidas y en el Congreso de Estados Unidos, lugares en donde Powell, Rice, Tenet y el propio Bush, brillaron por las inconsistencias y contradicciones de sus argumentos. De la misma manera, la Comisión de Control, Verificación e Inspección (UNMOVIC) a cargo de Hans Blix, nunca pudo encontrar las supuestas ADM, ni siquiera halló pruebas fehacientes el grupo a cargo de Hans Blix.

Más allá de la violación de la normatividad internacional y la falta de respeto a la ONU por parte de Washington, dicho sea de paso, la institución más exitosa y guardiana del orden internacional, luego de la Segunda Guerra Mundial, lo que se deduce, es un gran cambio en la arquitectura de la política estadounidense, que se perfiló de una forma agresiva, que sólo se entendería a partir de los postulados de realistas clásicos como Morgenthau, Hobbes o Machiavello, en donde la lucha por el poder, a cualquier precio, es lo más importante. La aparente inestabilidad del orden mundial, generada por el unilateralismo de EE.UU., podría sustentarse en lo que Kenneth Waltz llamara luego del 11-s: «un gran desbalance de poder, desde el ocaso de la URSS, una gran proliferación de ADM, y una permanente crisis mundial en donde EE.UU. está envuelto». ${ }^{1}$

\footnotetext{
${ }^{1}$ Waltz 2002. Citado en Kennedy-Pipe 2006, 543.
} 
No obstante, si se revisan con atención varios antecedentes, las acciones de Washington, obedecen a una estrategia coherente con los intereses de unos grupos particulares, que luchan entre si por la acumulación de dinero y poder y que han sido capaces, de fortalecer el unilateralismo político de Bush, con un discurso agresivo en donde se era aliado o enemigo, menospreciando a la ONU y la OTÁN y considerando como una traición la posición crítica de Alemania y Francia. ${ }^{2}$

Ahora bien, sería muy ingenuo pensar que el 11-s, es el único factor que desencadenó la nueva postura de la administración Bush, es lógico que hizo las veces de catalizador, pero las directrices diplomáticas de EE.UU., ya se estaban delineando a través de un staff político, al que catalogaron como neocons ${ }^{3}$, los cuales definirían la cultura estratégica que Berger, ${ }^{4}$ define como una realidad negociada entre élites de política exterior, lo cual concuerda con lo expuesto por Izquierdo: "Cualquier proceso de democracia representativa es una muestra clara de acumulación de poder, cedido por la mayoría de la población, en manos de unas elites que así tienen acceso al Estado como recurso». ${ }^{5}$ En el mismo orden, Reynolds manifiesta que el interés de un subgrupo nacional, como por ejemplo los dueños de las compañías de petróleo, pueden influir en que EE.UU., mantenga una posición dominante en Oriente Medio, y expresa que el interés nacional de un país, no se puede concebir sin la conexión de intereses de esos subgrupos ${ }^{6}$, de la misma manera Samir Amín manifiesta, que la región es importante para los intereses de Washington por tres razones: el petróleo, su ubicación geopolítica estratégica en el corazón de lo que él denomina "viejo mundo" y el hecho adicional de que constituye parte de la base del sistema mundial, 7 veamos cómo se articula la estrategia.

Se debe precisar que Oriente Medio, es considerado por algunos teóricos, como una abstracción artificial del siglo XIX, impuesta por las autoridades británicas de la época y es sólo hasta el

${ }^{2}$ Barbé 2006, 29-31. La autora se aproxima a la posición de Waltz, argumentando que la crisis del orden mundial se ha generado por tres factores: i) cambios en la estructura del sistema internacional, ii) diferente evolución de las sociedades estadounidense y europea y, iii) giro en la política exterior de Bush.

${ }^{3}$ El equipo original de «neocons» esta compuesto por: Collin Powell, Secretario de Estado; Donald Rumsfeld, Secretario de Defensa; Paul Wolfowitz, Diputado del Secretario de Defensa; Elliot Abrams, Asistente Especial del Presidente y del Consejo de Seguridad Nacional; Paula Dobriansky, Subsecreataria de Estado para Asuntos Internacionales; Richard Armitage, (US) Diputado del Secretario de Estado; Robert Zoellick, (US) Representante de Comercio; John Bolton, (US) Subsecretario de Estado para el Control de Armas y Seguridad Internacional y Richard Perle. Otros tenían fuertes nexos con el sector petrolero, como por ejemplo el Vicepresidente Dick Cheney (Harken), y la asesora de seguridad Condolezza Rice (Chevron).

${ }^{4}$ Berger 1988, 11-12. Citado en Lantis 2002, 107.

${ }^{5}$ Izquierdo 2008, 50.

${ }^{6}$ Reynolds 1994, 40.

${ }^{7}$ Amin 2006, 81. 
fin de la II Guerra Mundial, que los políticos empiezan a usarlo sin tener en cuenta sus límites geográficos. ${ }^{8}$

\section{GEOPOLÍTICA DEL PODER, ANTECEDENTES DURANTE EL SIGLO XX.}

La geopolítica en términos de Parker, es «el estudio espacial de las relaciones entre estados y las implicaciones de estas relaciones, para la forma del mapa político como un todo y puesto al servicio del Estado» ${ }^{9}$ En este sentido Irak siempre ha sido un foco de atención para analistas y gobernantes de Washington, incluso desde 1911, cuando se fundó la Turkish Petroleum Company (TPC) como una sociedad entre Alemania, Holanda y Gran Bretaña. En esa época, Irak aún hacía parte del Imperio otomano, liderado por los turcos, el cual sería derrotado en 1917 por los ingleses, quienes luego controlarían Palestina y Jordania.

En 1923 la TPC dio paso a la formación de la Irak Petroleum Company (IPC), empresa en la que participaban accionistas ingleses y franceses, quienes a partir de ese momento, contarían con la presencia de las compañías antecesoras de la Exxon y Mobil estadounidenses. Rápidamente, la IPC empezó a generar grandes utilidades. A cambio de reconocer la soberanía de Irak, Gran Bretaña exigió que se le concediera la explotación del petróleo por medio de la IPC, petición que fácilmente consiguió, debido a que Nuri As - Said, gran amigo del legendario británico Lawrence de Arabia, ocupaba el cargo de primer ministro, mientras que en el trono se encontraba el rey Faisal.

En 1952, se renovó el tratado que favorecía a la IPC y sus políticas pro-occidentales, basadas en la represión y el establecimiento de relaciones con EE.UU.. Esta situación dio lugar a varios

\footnotetext{
${ }^{8}$ Gerges 1991, 209. Para este autor, el problema de la definición no es sólo académico, también es ideológico y político. Igualar al Oriente Medio con una entidad particular (la región Pan-Árabe) o una comunidad Islámica (la umma Musulmana) conlleva implicaciones políticas. Esta caracterización implica el rechazo de un discurso académico que usa el concepto «Medio Oriente» como algo garantizado. Algunos teóricos árabes califican el concepto como producto del colonialismo e imperialismo occidental, y lo sustituyen por el de un subsistema regional árabe el cual, desde su punto de vista, representa de forma más sensata las relaciones e interacciones de los actores en el área; veamos en palabras de Gerges la dificultad que conlleva definir los límites de la zona: «Some analysts extend the 'Middle East' to include Iran, Afghanistan, Pakistan, Turkey, the Maghrib, Israel and, of course, the Arab states. Other observers equate the 'Middle East' with the Arab world to the exclusion of Iran, Pakistan, Afghanistan, Turkey and even Israel. To some, the 'Middle East' means the Islamic world with its vast territories, including Morocco on the west and Bangladesh, India and Russian Turkestan on the east. Still others recognize that the 'Middle East' may be more a psychological than a geographical area. Anthropologists define the region as a culture area extending from Morocco to Timbuktu, from Russian Turkestan to West Pakistan. It should be apparent by now that there is no accepted formula to delimit the region. Definitions vary depending on the analysts' research designs».
}

${ }^{9}$ Parker 1994, 170. 
golpes de Estado, hasta que en 1958, en medio de una revolución, fueron asesinados,, el rey y el primer ministro. Se proclamó el nacimiento de la República de Irak, bajo el mando del general Abd al-Karim Qasem, quien sería derrocado 10 años más tarde en 1968, a raíz de un golpe propinado por dos oficiales del partido Ba'ath: Ahmed Asan al-Bkr y Saddam Hussein, quienes de inmediato nacionalizaron la IPC. ${ }^{10}$

Posteriormente, en 1979, los problemas de salud de su compañero y las oscuras maniobras políticas de Hussein, le permitieron hacerse el poder de Irak, tras la renuncia de Asan Al-Bakr. Con su formación militar y sus delirios por fortalecer a su país, como la nación del Mundo Árabe más poderosa, Hussein comenzó a utilizar los recursos del petróleo de forma estratégica. Gracias al boicot propiciado por los países árabes desde las guerras de 1973, la renta de Irak se incrementó, pasando de US \$572 millones en 1972, a US \$ 5.700 millones en 1974.

Oriente Medio, se consolidó como la región petrolera más importante del mundo, razón fundamental para que la política exterior de EE.UU., mostrara un gran interés sobre Irak después de las crisis energéticas mundiales de 1973 y 1979, cuando la falta de combustibles generó un caos en los mercados mundiales. En 1979 el catedrático R.K. Ramazani de la Universidad de Virginia, publicó en la revista Foreign Affairs, un artículo titulado "Security in the Persian Gulf», en cual se preguntaba si EE.UU., tendría la capacidad de mantener la seguridad en esa región, y destacaba que éste era un cuestionamiento constante en la mente de los políticos de Washington en ese entonces. ${ }^{11}$

Terminada la Guerra Árabe - Israelí de 1973, la percepción de Irán como «país protector de la seguridad del Golfo» se fortaleció por la ayuda brindada por EE.UU. a ese país, luego de que en 1971, Gran Bretaña tomara la decisión de retirar sus tropas de la zona del Suez y del Golfo Pérsico. El limitado acceso a las aguas del Golfo, otorgaba una posición privilegiada a Irán, nación que era considerada como el "gran pasaje», debido al Estrecho de Hormuz, a través del cual pasaba alrededor del $57 \%$ del petróleo rumbo a los mercados internacionales.

Pero en 1979, con la caída del Alí Reza Pahlevi, más conocido como el Shá de Irán, un fiel aliado de los intereses de Washington y el intempestuoso ascenso al poder del Ayatollah Homeini,

\footnotetext{
${ }^{10}$ El coronel Abd al-Salam Aref colaboró a Qasem en la revolución, pero este le envió a prisión. Cuando Qasem fue ejecutado el 9 de febrero de 1963, Aref tomó las riendas del poder en Irak, pero murió en un misterioso accidente de helicóptero, el 13 de abril de 1966. Le sucedió su hermano Abd al-Rahman Aref, que se caracterizaba por una notable falta de autoridad y liderazgo. Sólo logró sobrevivir políticamente dos años, hasta la Ilegada del partido Ba'ath.

${ }^{11}$ Ramazani 1979, 821-828.
} 
un líder religioso radical, que se declaraba opuesto a los valores occidentales, Irán quedó bloqueada para los planes geoestratégicos de EE.UU., y pasó de aliado a enemigo.

Mientras tanto, Hussein importaba el $65 \%$ de los alimentos y compraba armas a Gran Bretaña, Francia y la URSS, bajo la mirada complaciente de Washington, convirtiéndose en el objetivo más importante para la política exterior estadounidense en la zona norte del Golfo Pérsico. La benevolencia hacia el régimen de Hussein, le permitió a Irak organizar un ejército de aproximadamente un millón de hombres, el más grande del mundo árabe y uno de los cuatro más numerosos del planeta, con el propósito de iniciar una guerra contra Irán en 1980, que duró ocho años, y dejó un saldo de casi un millón de muertos. ${ }^{12}$

El apoyo económico y militar que recibió durante el lapso de la guerra Irán-Irak, le facilitó a Saddam Hussein, concebir una estrategia expansionista que incluía a Kuwait y posteriormente, a Arabia Saudita, una ambición de tipo «imperial» que Washington no estaba dispuesta a permitir, más aún, cuando lo que estaba en juego era el acceso a los recursos energéticos, y la seguridad de la región más rica del mundo en yacimientos petrolíferos. El 2 de agosto de 1990, Hussein decidió invadir a Kuwait, convirtiéndose en el nuevo enemigo de EE.UU. en la región. ${ }^{13}$

No obstante, se debe tener en cuenta que de 1960 a 1980, los EE.UU., hicieron seis intentos en medio de una posible guerra regional, para detener: el uso de la fuerza propia, la llegada de fuerzas externas y, el despliegue de sistemas de armas avanzados. Según la autora en los seis casos la disuasión falló, veamos:

\section{Tabla 1.}

\section{Casos en los que falló la disuasión extendida de EE.UU. en O.M.}

1. Prevenir un ataque egipcio sobre Arabia Saudita, durante las primeras fases de la Guerra en Yemen

2. Detener la intervención soviética en los días finales de la Guerra Árabe - Israelí en 1967

3. Detener el envío de personal de combate a Egipto durante su guerra de fricción con Israel a principio de 1970

4. Detener a la URSS y a Egipto sobre el avance de un sistema anti-misiles en la zona del Canal del Suez

\footnotetext{
${ }^{12}$ Revista Cambio. (Bogotá) 2003. «La fiebre del Oro Negro». (508): 25-27.

${ }^{13}$ Un excelente trabajo sobre el comportamiento, las decisiones y el ambiente geopolítico que llevaron a Saddam Hussein a la Guerra del Golfo de 1991, a partir de sus percepciones, y bajo una mirada constructivista se encuentra en: Freij, Hanna. 2001. "Self-Image and Role Definition as a Cause of War: Saddam Hussein 1988-90». The Journal of Conflict Studies. 21(1):101-121.
} 
5. Detener un ataque sirio contra Jordania en septiembre de 1970

6. Detener la intervención militar soviética en los últimos días de la guerra de octubre de 1973.

Fuente: Gross Stein 1988, 146. Elaboración propia

Por su parte, Haass ${ }^{14}$ establece cinco eras en Oriente Medio, que resultan útiles para complementar la información de la tabla anterior y acercarnos con más criterio a la actualidad, en donde el conflicto interno que se ha gestado en Irak, que se puede catalogar de guerra civil, es uno del temas más complejos para predecir futuros escenarios, veamos ahora la propuesta de Hass:

\begin{tabular}{|l|l|}
\hline \multicolumn{2}{|l|}{ Períodos en Oriente Medio } \\
\hline \multicolumn{1}{|l|}{$\begin{array}{l}\text { Guerra Mundial } \\
\text { 2. Colonial }\end{array}$} & $\begin{array}{l}\text { Fallecimiento del Imperio Otomano, levantamiento de la República } \\
\text { Turca y la división de los despojos de esa guerra entre los } \\
\text { vencedores europeos. }\end{array}$ \\
\hline 3. Guerra Fría & $\begin{array}{l}\text { Dominada por Francia y Gran Bretaña, finaliza cuatro décadas } \\
\text { después cuando la II Guerra Mundial, había agotado a las potencias } \\
\text { europeas, el nacionalismo árabe había crecido y EEUU y la URSS } \\
\text { empezaban a usar sus zonas de influencia. } \\
\text { "Who rules the Near East rules the World, and he who has interest } \\
\text { in the World is bound to concern himself with the near east"; } \\
\text { escribió el historiador Albert Hourani., quien vio el fin de la era } \\
\text { colonial con en 1956 con la Crisis del Canal del Suéz, y el comienzo } \\
\text { de la Guerra Fría en la región. }\end{array}$ \\
\hline 4. Dominio total de & $\begin{array}{l}\text { Dominio total del EE.UU. en la región, la liberación de Kuwait, el } \\
\text { estacionamiento de fuerzas terrestres y aéreas en la península } \\
\text { arábiga y una diplomacia activa, tratando de resolver el conflicto } \\
\text { entre Israel y Palestina. }\end{array}$ \\
\hline 5. iEE.UU? & $\begin{array}{l}\text { Contradicciones, menos influencia de EE.UU. refleja límites de su } \\
\text { poder. } \\
\text { Posibles presiones de UE sobre la cuestión de Palestina. } \\
\text { Irán podría convertirse en un estado más poderoso. } \\
\text { Irak podría estar en problemas algunos años más. }\end{array}$ \\
\hline
\end{tabular}

Fuente: Haass 2006. Elaboración propia

\footnotetext{
${ }^{14}$ Haass 2006.
} 
Con los atentados del 11-s, la política exterior de EE.UU., asumió una nueva forma de acción política, producto de nuevas circunstancias que desde Washington son vistas en términos tradicionales de seguridad, muy cercanos a una aproximación realista en la que confluyen países poderosos y débiles, proliferación de armas y amenazas a la seguridad como el terrorismo. Estos aspectos han hecho que el gobierno de George W. Bush, haya desarrollado una estrategia que en palabras del propio presidente estadounidense «no está abierta al debate». ${ }^{15}$

La decisión de llevar a cabo un ataque preventivo en Irak, generó reacciones encontradas en diversos sectores de la opinión pública; algunos intelectuales manifestaban que la guerra estaba motivada por algo más que el terrorismo: en primer lugar, ubicaron el interés por el petróleo y en segundo lugar, la obtención del control hegemónico de Oriente Medio. Otros argumentos que también consideraron importantes, fueron el deseo de venganza de Bush padre y el apoyo de Washington a Israel, ${ }^{16}$ pero es el libro de Stephen Walt y John Mearsheimer The Israel Lobby, el que más revuelo ha causado en la opinión pública estadounidense, ya sido fuertemente contestado por la comunidad académica y por sectores judíos en EE.UU. ${ }^{17}$

Los ataques preventivos, ${ }^{18}$ se mostraron ante la opinión pública como la mejor forma de derrocar el régimen de Saddam Hussein, argumentando que el riesgo de no actuar a tiempo, contra amenazas terroristas, traería graves consecuencias para la seguridad nacional de EE.UU., y de los demás países del mundo desarrollado. ${ }^{19}$ No se puede olvidar que durante casi 80 años, la política estadounidense hacia Medio Oriente, ha estado encaminada a cumplir metas específicas: asegurar las reservas de petróleo y establecer el control de la región, metas que continúan creando problemas y resistencia, ${ }^{20}$ es más, la mayoría de trabajos sobre el tema poseen un toque

\footnotetext{
${ }^{15}$ Hastings Dunn 2003, 284.

${ }^{16}$ Personajes reconocidos internacionalmente se manifestaron en contra de la Guerra de Irak 2003: Baltasar Garzón Real, magistrado español, Norman Mailer, novelista estadounidense ganador del Premio Pulitzer, Jhon Le Carré, escritor británico, Carlos Fuentes, novelista mexicano, Jeffrey Sachs, profesor titular de economía y director de Earth Institute, Columbia University, Robert Fisk, periodista irlandés del diario The Independent y Juan Goytisolo, escritor español. Una compilación de sus artículos se encuentra en: Revista Nueva Gaceta, (Bogotá) 2003. «Voces contra la guerra a Irak». (6):6-19.

${ }^{17}$ El trabajo se presentó primero en forma de ensayo en London Review of Books en 2006, Mearsheimer, John J. and Walt, Stephen. The Israel Lobby, London Review of Books, Volume 28 Number 6, March 23, 2006. Luego el libro fue publicado en 2007 como: The Israel Lobby and U.S. Foreign Policy. New York. Farrah, Strauss, and Giroux.
}

${ }^{18}$ Existe un debate sobre si los ataques son preventivos o anticipatorios, pues en inglés preemptive en ocasiones es reconocido como anticipatorio, esta es una de las definiciones: «Preemptive: military intended to prevent attack: intended to eliminate or lessen an enemy's capacity to attack».

${ }^{19}$ Hastings Dunn Op.cit., 288.

${ }^{20}$ Watkins $1997,1$. 
de pesimismo sobre la relativa pérdida de EE.UU., en la actual intervención; ${ }^{21}$ veamos a continuación si son tan adecuados.

\section{EL FRACASO DE LA RECONSTRUCCIÓN Y EL ÉXITO DEL CONTROL DE RECURSOS.}

Atrás quedaron las imágenes de la caída de la estatua de Hussein, y las palabras de Bush el 2 de mayo de 2003, cuando en una escena fílmica, a bordo del portaviones Abraham Lincoln, proveniente del Golfo Pérsico con cinco mil hombres, manifestara: «Esta batalla es una victoria en la guerra contra el terrorismo, la fase bélica ha terminado, peleamos por la causa de la libertad y la paz del mundo». Esta era una clara estrategia de medios, pues la guerra que había iniciado el 19 de marzo ese mismo año, habría de prolongarse por cinco años más, sin que a la fecha, exista un panorama claro para la situación interna del país.

Rápidamente el manejo administrativo en Irak se convertiría en un desastre ${ }^{22}$ la promesa de la transición político-militar, fue un objetivo que nunca consiguió Paul Bremer quien coordinó la (CPA) Coalition Provisional Authority (por sus siglas en inglés), quien además cometió graves errores de cálculo, desconociendo por completo la compleja estructura de clanes iraquí, y la herencia de la imagen negativa de EE.UU. ante un pueblo chí́ta y kurdo que le asociaba con el apoyo al brutal régimen de Hussein durante su dictadura a partir de la década de 1980.

Su larga lista de errores sociales, militares y políticos demostraron su incapacidad para manejar el reto que implicaba estar en un Irak herido y devastado por años de guerra. Las primeras incompatibilidades se presentaron con los generales a cargo de las tropas. El mayor general

${ }^{21}$ Estos son los títulos: CHANDRASEKARAN, Rajiv 2006. Who Killed Irak. Foreign Policy. (Sep-Oct). p.36-43. Dobbins, James 2007. Who lost Iraq? Lessons from the debate. Foreign Affairs. 86 (5): 61-74. Fearon, James. 2007. U.S. Can 't Win Iraq's Civil War.Foreign Affairs. 86 (2): 2-15.

${ }^{22}$ Fearon 2007. En su artículo manifestaba: «La tasa de muertos en Irak -más de 60 mil en los últimos tres añosubica el conflicto al lado de otros descritos como guerras civiles. (por ejemplo, Argelia, Colombia, Guatemala, Perú y Sri Lanka). La cifra conservadora de 60 mil muertes, podría ubicar a la guerra civil iraquí en el noveno lugar de mortalidad en este tipo de conflictos desde 1945. De la misma manera, Fearon argumenta que las guerras civiles son difíciles de terminar. Su promedio de duración desde 1945 ha sido de 10 años, y la mitad ha durado más de 7 años. Su duración en el resultado de la forma en que se combate, los grupos rebeldes usas tácticas de guerrilla, operan en regiones rurales de países postcoloniales con un sistema administrativo, político y policial débil. Las guerras civiles como la estadounidense, con ejércitos convencionales y frentes definidos, que se enfrentan entre sí son inusuales; son más comunes como las tácticas de guerrilla que se usan en los países ya nombrados como Argelia, Colombia, Sri Lanka y el sur oriente de Sudán. Estos casos ilustran cómo la guerrilla rural puede ser una táctica robusta, que con un relativamente pequeño número de rebeldes se puede obtener el control parcial de grandes partes de territorio durante muchos años, a pesar de las costosas y brutales campañas militares en contra de ellos». 
David Paetreus, advirtió a Bremer del peligro que implicaba despedir a personal del partido Baath de sus trabajos del gobierno, entre los que se encontraban asesores del ministro de salud, 15 mil profesores y viejos soldados del partido que eran miembros honorarios, luego de pasar años como prisioneros de guerra en campos iraníes. ${ }^{23}$ De la misma manera, excluir a los Baathistas del ejército era algo muy peligroso, porque fácilmente caerían en la insurgencia. Este tipo de debates entre la administración civil y militar se prolongarían durante 15 meses, lapso en el que se fue delimitando el clima de inseguridad y peligro que continua hasta la actualidad.

En el mismo orden, otro error de cálculo fatal tiene que ver con el menosprecio al líder chí́ta Moqtada al-Sadr, heredero del ayatolá Muhammad Sadiq al-Sadr, quien fue asesinado por el régimen de Hussein junto con dos hijos en 1999. Bremer, no le permitió un escaño en el recién constituido parlamento y además ordenó el cierre del periódico Al Hawzam ${ }^{24}$ el 28 marzo de 2004, bajo el argumento de que se utilizaba para difundir arengas en contra de Washington.

Esta fatal decisión, generaría el levantamiento de la insurgencia chiíta en Bagdad y en el sur del país, a través de la milicia leal a al-Sadr, conocida como Ejército Mahdi, los cuales han combatido ferozmente desde entonces. ${ }^{25} \mathrm{El}$ comandante del ejército estadounidense en aquél entonces, Ricardo Sánchez nunca estuvo de acuerdo con la persecución a los shí́tas, pero Bremer era quien mandaba. ${ }^{26}$ Otros errores estratégicos, se cometerían al no abrir los mercados financieros, hasta pasados cinco meses de la intervención, cuando lo que deseaban los iraquíes era reestablecer su economía lo más pronto posible, problemas parecidos de mala gestión y confusión ocurrieron con la red hospitalaria, lo cual provocó graves consecuencias directas en la población. El último informe del Internacional Crisis Group, del 7 de febrero de 2008, sobre Oriente Medio, uno de los think tanks más influyentes resume el caos que se vive en el país, así:

\footnotetext{
${ }^{23}$ Chandrasekaran 2006, 37.

${ }^{24}$ Uno de las argumentos de Bremer para el cierre del periódico fue un reportaje en febrero que decía «Bremer sigue los pasos de Saddam», en donde se le acusaba de dejar morir de hambre deliberadamente al pueblo iraquí.

${ }^{25}$ Dos días antes del cierre del periódico los marines estadounidenses habían matado a 15 iraquíes durante una incursión. Esa misma semana, y luego del cierre del periódico, cuatro contratistas de seguridad fueron asesinado y mutilados, sus cuerpos luego fueron colgados en un puente sobre el Río Éufrates. Estos hecho desencadenarían una de las más fatales matanzas de civiles por parte del ejército de EE.UU. cuando dos mil marines atacaron la ciudad de Faluya, este hecho catapultaría la imagen de al-Sadr frente las sunitas, quienes desde entonces lo ven como algo más que un simple agitador. Para más información y crónicas periodísticas sobre estos hechos véase el libro de CHANDRASEKARAN, Rajiv: Vida Imperial en la Ciudad Esmeralda. En el interior de la Zona Verde de Bagdad. Ed. RBA. 2008.
}

${ }^{26}$ Chandrasekaran, Op cit., 40. 
"The Sadrists appeared on a steady rise in 2006 and early 2007. They controlled new territory, particularly in and around Baghdad, attracted new recruits, accumulated vast resources and infiltrated the police. But as the civil war engulfed much of the country, Iraqis witnessed the Sadrists' most brutal and thuggish side. Their increasingly violent and undisciplined militia, the Mahdi Army, engaged in abhorrent sectarian killings and resorted to plunder and theft. Militants claiming to be Mahdi Army members executed untold numbers of Sunnis, allegedly in response to al-Qaeda's ruthless attacks, but more often than not merely because they were Sunnis». ${ }^{27}$

De la misma manera, Amnistía Internacional, resume la situación caótica al inicio de su último informe Carnage and Despair, Iraq Five Years $\mathrm{On}^{28}$, y hace énfasis, en el número de desplazados y refugiados en Siria y Jordania:

«Five years after the US-led invasion that toppled Saddam Hussein, Iraq is one of the most dangerous countries in the world. Hundreds of people are being killed every month in the pervasive violence, while countless lives are threatened every day by poverty, cuts to power and water supplies, food and medical shortages, and rising violence against women and girls. Sectarian hatred has torn apart families and neighborhoods that once lived together in harmony».

"As the sectarian violence became more acute, a deepening humanitarian catastrophe began to unfold. At least four million people have become displaced - nearly 15 per cent of Iraq's estimated total population of 27 million. According to the UN High Commissioner for Refugees (UNHCR), about two million people are living in Syria and Jordan,2 while at least 2.2 million others are displaced inside Iraq and living in appalling conditions».

Por su parte, el Comité Internacional de la Cruz Roja, corrobora los argumentos que exponen las organizaciones anteriores:

« Cinco años después del desencadenamiento de la guerra en Irak, la situación en la mayor parte del país, sigue siendo desde el punto de vista humanitario, una de las más críticas del mundo. El conflicto impide que millones de iraquíes, tengan suficiente acceso a agua potable y a servicios de salud y de saneamiento adecuados. Además de la actual crisis, perduran los efectos de los anteriores conflictos armados y de los años de sanciones económicas».

«A pesar de ciertas mejoras por lo que respecta a la seguridad en algunas zonas, la violencia armada sigue teniendo desastrosas consecuencias. Las hostilidades, continúan causando la muerte de perso-

\footnotetext{
${ }^{27}$ Crisis Group 2008.

${ }^{28}$ Amnesty International 2008.
} 
nas civiles y muchos heridos no reciben una atención médica apropiada. Para millones de personas, el abastecimiento de agua es insuficiente y de mala calidad, debido a la falta de mantenimiento de las plantas de abastecimiento de agua y de tratamiento de aguas residuales, así como al escaso número de ingenieros». ${ }^{29}$

Luego de lo anterior, se puede evidenciar que el manejo del conflicto interno y la reconstrucción iraquí, han sido producto de una secuencia de errores, con nefastos resultados para la población local, pero en contraposición se puede analizar el manejo que se ha dado a los recursos energéticos y al tema de la seguridad en la región. Washington ha desarrollado un despliegue táctico de tropas en la zona, que si bien tienen problemas con el caos interno del país, se encuentran ahora más cerca de estados considerados hostiles por Washington como Irán, Siria y Pakistán. Asimismo, se está más cerca del otro escenario de lucha clave en la catalogada «Guerra contra el Terror»: Afganistán. Se puede agregar que esta ubicación le permite estar más cerca de China e India, potencias emergentes a las que se debe prestar atención, según algunos académicos realistas como Mearsheimer. ${ }^{30}$

Por lo tanto, el descuido del orden público en Irak, y el control de recursos energéticos, son aspectos opuestos de una misma geoestrategia, pero que también tienen fuerte conexión con la explotación de crudo y la vigilancia de su transporte a través del Golfo Pérsico ${ }^{31}$, de otra forma no se explicaría la inversión en infraestructura en la zona, por ejemplo la gran embajada que se está construyendo; ${ }^{32}$ veamos qué nos dice el último informe de la OPEP respecto a la exportación de petróleo durante 2007:

«Mercado mediterráneo. Los diferenciales de los Urales estuvieron bajo presión de las altas exportaciones iraquíes...»; «...Sin embargo, el sentimiento de llegar a estar bajo presión de la venta de petróleo iraquí a partir del crecimiento constante de su mercado en el norte.... ${ }^{33}$ «El incremento en la refinería ha generado un fuerte impacto en las reservas de petróleo, así como el crecimiento de crudo procedente de Irak».34

${ }^{29}$ ICRC 2008.

${ }^{30}$ Mearsheimer 2001. 361. Para este autor (realismo ofensivo) China es la única amenaza para la hegemonía de EE.UU.

${ }^{31}$ Las siguientes son unas estimaciones sobre la importancia del petróleo en Oriente Medio: El 65\% de las reservas mundiales del petróleo se encuentran en el Medio Oriente, las 2/3 partes de dichas reservas se encuentran en la región del Golfo Pérsico, en donde el primer lugar (en reservas) lo tiene Arabia Saudita con 261 billones de barriles, equivalente al 25\%, y el segundo lugar, lo ocupa Irak con 110 billones de barriles, equivalente al $12 \%$ dela oferta mundial. (Véase Figura 2). Actualmente el $25 \%$ de la producción mundial proviene de esa región. En contraposición, el subsuelo de EE.UU. sólo cuenta con el $2 \%$ de las reservas conocidas en el mundo, mientras dicho país consume más del $25 \%$ de la producción mundial anual.

${ }^{32}$ Para una reseña de la construcción de la nueva embajada de EE.UU. en Bagdad «la mayor que nunca ha visto el mundo», véase: Loeffler, Jane. 2007. «Búnker en Bagdad». Foreign Affairs en Español. (Oct - Nov) p.62-66.

${ }^{33}$ OPEP 2008, 6.

${ }^{34}$ Ibíd., p.43. 
Tabla3.

\begin{tabular}{|l|l|}
\hline \multicolumn{2}{|l|}{ Producción de la OPEP (Diciembre de 2007) } \\
\hline (en millones de barriles diarios por día, de acuerdo a fuentes secundarias) \\
\hline Arabia Saudita 8,98 & Angola 1,81 \\
\hline Irán 3,90 & Libia 1,74 \\
\hline Kuwait 2,55 & Argelia 1,40 \\
\hline EAU 2,53 & Indonesia 0,85 \\
\hline Venezuela 2,39 & Qatar 0,84 \\
\hline Irak 2,33 & Ecuador 0,51 \\
\hline Nigeria 2,18 & TOT AL 31,99 \\
\hline
\end{tabular}

Fuente: Urtr. Monthıy Uıl Market Keport. January zUU8. Sumarı. p.১I. tlaboracıon propıa.

Figura 1.

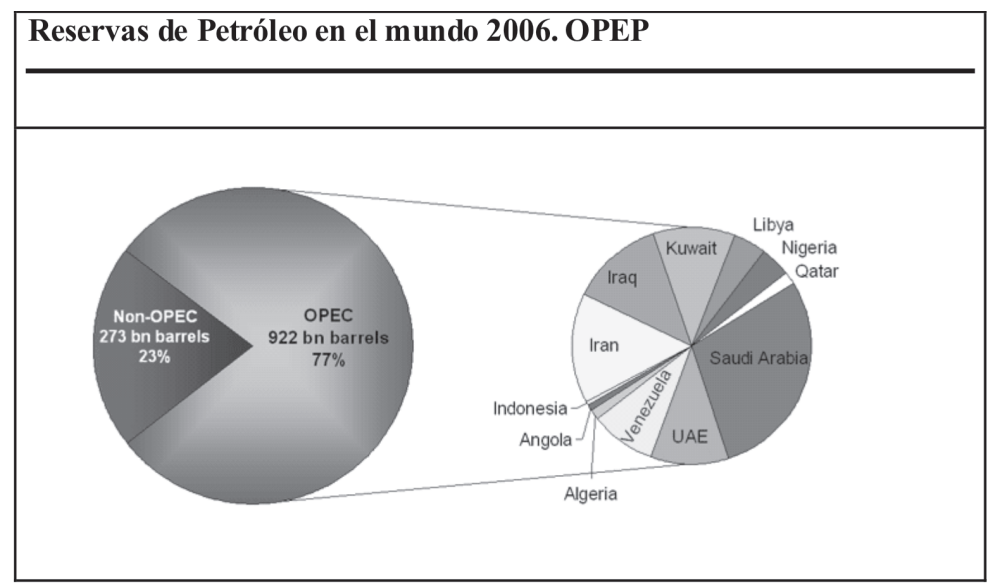

Fuente: OPEP. http://www.opec.org/home/PowerPoint/Reserves/OPEC\%20share.htm

Como se observa en la tabla 3, Irak ha aumentado su producción, para diciembre de 2007, a 2.3 $(\mathrm{mbd})$ ¿por qué no aclarar que se trata de millones? millones de barriles por día, superando a países como Argelia, Angola, Ecuador, Indonesia, Libia, Nigeria, Katar, y está muy cerca de Emiratos Árabes Unidos y Venezuela. Lo anterior concuerda con los vaticinios que hiciera el ministro de petróleo iraquí Thamir Ghadhban en mayo de 2003, cuando la ONU levantó las sanciones al país, manifestó que para junio de ese año se comenzarían a exportar 700 mil barriles diarios hacia EE.UU. El 20 de junio de 2003, la cifra de barriles diarios exportados, era de 1.5 (mbd), es decir, (el $0.8 \%$ de su capacidad de producción), esa cifra ahora es mayor: 2.33 (mbd). 
El control del petróleo iraquí es paradójico, a pesar de que la explotación en gran medida es realizada por compañías estadounidenses, el precio se ha elevado a cifras que rondan los 100 dólares, lo cual ha incrementado el poder de uno de los grandes rivales de Washington en la región: Irán, país que de seguir la tendencia actual de crecimiento económico, se puede convertir en uno de los estados árabes más poderosos, con una gran influencia sobre grupos como Hamas y Hezbollah, a los cuales utiliza para rehacer su imagen y para fortalecer el shiísmo y alejarlo del sunismo, tal y como se esta viendo en Irak en la actualidad.

\section{CONCLUSIÓN.}

El 19 de marzo de 2008 se cumplieron 5 años de la intervención, muchas críticas se han hecho al gobierno de EE.UU., pero Bush, ha manifestado ante los medios que pese al alto costo económico (500 mil millones de dólares) y de vidas, (3.990 soldados muertos, más personal civil), ${ }^{35}$ la invasión ha valido la pena, argumenta además, que retirarse ahora fortalecería a Irán y proveería de dinero a Al-Qaeda, para adquirir armas de destrucción masiva con el propósito de atacar a EE.UU, ${ }^{36}$ afirmaciones que paradójicamente pueden ser ciertas, debido a los errores administrativos que inició Bremer.

En ese sentido, problemas tales como: corrupción oficial y no oficial, vigilancia esporádica, mala conducta policíaca, lucha de milicias, problemas políticos, fatiga económica, separatismo regional, incompetencia gubernamental, criminalidad desenfrenada, conflicto religioso, fuerza de empresarios políticos anti-americanos y retórica anti-israelita, serían efectos colaterales de los enfrentamientos modernos, más aún, un reciente estudio de Meter Fearer y Christopher Gelpi ha calculado que en promedio los americanos, apoyarían la muerte de 6,861 soldados con tal de Ilevar la democracia al Congo, ${ }^{37}$ lo cual haría suponer que las muertes de Irak, se

\footnotetext{
${ }^{35}$ El último informe de Amnistía Internacional sostiene que la cifra exacta de civiles muertos en el país, desde la invasión en marzo de 2003 es desconocida. Thw World Health Organization (WHO) y el Ministerio de Salud Iraquí, liderado por el Primer Ministro Nuri al-Maliki, han aceptado la cifra aparentemente. A finales de 2007, listas publicadas por investigadores estadounidenses de la publicación médica Lancet se aproximaban a 601.027. De acuerdo a la Misión de Asistencia para las Naciones Unidas de Irak (UNAMI) 34.452 personas fueron asesinadas y otro miles heridas. La violencia sectaria incrementó a partir del 22 de febrero de 2006 cuando fue destruida la mezquita chí́ta de al-Askari en la ciudad de Samarra.

${ }^{36}$ Bush Defends Iraq War in Speech. En The New York Times. «Removing Saddam Hussein from power was the right decision," President Bush said at the Department of Defense. También manifestó: "Los éxitos que estamos viendo en Irak son innegables, y a pesar de ello algunos en Washington siguen pidiendo una retirada. Los críticos de la guerra no pueden argumentar en forma verosímil que estamos perdiendo en este país, por lo tanto dicen que la guerra cuesta demasiado. En los últimos meses, hemos escuchado cálculos exagerados de los costos de esta guerra". Disponible: http://www.nytimes.com/ Acceso 19 marzo 2008.
} 
pueden tolerar porque el objetivo es más grande e importante y también estos datos demuestran lo paradójica y extraña que puede ser la opinión pública estadounidense, tal y como sucedió con las guerras mundiales, Corea, Vietnam y la primera guerra del golfo en 1991.

En términos geoestratégicos la política de EE.UU. hacia Irak, se basa en un programa calculado, el cual tiene como principales objetivos el control de recursos energéticos y en el incremento de la presencia militar en la región, una región que como bien expone Amín, es vital para el sostenimiento de su supremacía y ha sido objeto de interés desde comienzos del siglo XX, cuando el mercado de hidrocarburos empezó a ser vital para el desarrollo del mundo Occidental. La invasión o campaña militar en Irak desde 2003, ha sido una victoria parcial, se tienen los recursos energéticos y la presencia en la zona, el teme que tal vez no se debió intentar, tal y como lo dice Etzione, «[...] imponer la democracia a países que no estén preparados para ella como es el caso de Irak». ${ }^{38}$

El recrudecimiento de los enfrentamientos entre chí́tas, sunitas, kurdos y los estadounidenses, en el momento en que se escribe este artículo, explican en parte la complejidad de las «guerras civiles» en países donde se profesa el Islam, en un estudio diacrónico Duffy, ${ }^{39}$ manifiesta que en 42 guerras civiles religiosas desde 1940 hasta 2000, los gobiernos y rebeldes que se identificaron con el Islam, estuvieron involucrados en 34 conflictos $(81 \%)$, mucho más que aquellos identificados con otras religiones como los cristianos ( $21 \%-50 \%)$ o el hinduismo (7\%-16\%); y de la misma manera, las guerras civiles, esto podría corroborar la escalada del conflicto, pero este sería tema de un trabajo posterior al respecto.

\section{BIBLIOGRAFÍA}

Amin. Samir. 2006. «The Geopolitics of the New Collective Imperialism of the Triad: The Middle East, the EU, and US Imperialism». The European 's Borden. Global Imperialism in EU Expansion (Edited by: ENGELDI MAURO. Salvatore. Peter Lang Publishing, New York.

Amnesty International 2008. Carnage and Despair, Iraq Five Years On. Disponible: [http://www.amnesty.org/ es/library/asset/MDE14/001/2008/en/3f78611b-f1e9-11dc-adcd-cdafd0ab0dfe/mde140012008eng.pdf] Acceso 21 marzo 2008.

Berger, Thomas. 1988. Cultures of Antimilitarism: National Security in German and Japan (Baltimore, Md: John Hopkins University Press, 1988)

\footnotetext{
${ }^{37}$ Muller 2005, 44-54

${ }^{38}$ Etzione 2004, 101.

${ }^{39}$ Duffy 2007, 97.
} 
Chandrasekaran, Rajiv. 2006. «Who Killed Iraq». Foreign Policy:36.43.

Crisis Group 2008. Iraq's Civil War, the Sadrists and the Surge, Middle East Report N72, (7 February 2008). Disponible [http://www.crisisgroup.org/home/index.cfm?id=5286\&l=4] Acceso 19 marzo 2008.

Duffy Toft, Monica. 2007.Getting Religion? The Puzzling Case of Islam and Civil War. International Security.31(4):97-131.

Etzione, Emitai. 2004. «The War Against Terrorism and Saddam 's Iraq: Constrasting Designs». From Empire to Community. Palgrave. Macmillan.

Fearon, James. 2007. Iraq's Civil War. Foreign Affairs. (Marzo Abril).

Gerges, Fawaz A. 1991. The Study of Middle East International Relations: A Critique. British Journal of Middle Eastern Studies. 18(2):208-220.

Gross Stein, Janice. 1988. The Wrong Strategy in the Right Place.: The United States in the Gulf. International Security 13(3).

Hastings Dunn, David. 2003 «Myths, Motivations and «Misuderestimations» The Bush administration and Irak. International Affairs 79( 2):284.

Haass, Richard. 2006. The New Middle East. Foreign Affairs. (6):2-11.

ICRC 2008. Irak: No hay tregua en la crisis humanitaria. Disponible: [http://www.icrc.org/web/spa/sitespa0.nsf/ html/iraq-report-170308] Acceso 17 de marzo de 2008

Ken Booth and Tim Dunne, eds, Worlds in collision: terror and the future of global order (London: Palgrave, 2002).

Lantis, Jeffrey. 2002. Strategic Culture and National Security Policy. International Studies Review.

Mearsheimer, John. 2001. The Tragedy of Great Power Politics, Nueva York, W.W. Norton.

Muller, John.2005. «The Iraq Síndrome. Foreign Affairs. (84)6:44-54.

OPEP. 2008. Monthly Oil Market Report. January 2008. Disponible [http://www.opec.org/home/PowerPoint/ Reserves/OPEC\%20share.htm] Acceso 13 marzo 2008. 
Parker, Geoffrey. 1994. Political Geography and Geopolitics. En: Groom A.R.J. y Light Margot. Contemporary International Relations: a Guide to Theory. Pinter Publishers. UK.

Reynolds, P.A. 1994. An Introduction to International Relations. Longman Group. UK.

Ramazani, R.K. 1979. Security in the Persian Gulf. Foreign Affairs:821-828.

Watkins, Eric. 1997. «The unfolding US policy in the Middle East». International Affairs. 73(1).

Waltz, Kenneth. The Continuity of Internacional Politics. Boot and Dunne Eds. Worlds in Collision. p.543. Citado en Kennedy-Pipe, Caroline. (2006) «Apocalypse Now? Continuities or Disjunctions in World Politics». International Affairs 83(2). 